\title{
Theory of neuronal spike densities for synchronous activity in cortical feed-forward networks Sven Goedeke ${ }^{1}$, Tilo Schwalger ${ }^{2}$ and Markus Diesmann*1,2
}

\author{
Address: ${ }^{1}$ Bernstein Center for Computational Neuroscience, Albert-Ludwigs-University, 79104 Freiburg, Germany and ${ }^{2}$ Computational \\ Neuroscience Group, RIKEN Brain Science Institute, Wako-shi, Saitama 351-0106, Japan \\ Email: Markus Diesmann* - diesmann@brain.riken.jp \\ * Corresponding author
}

from Seventeenth Annual Computational Neuroscience Meeting: CNS*2008

Portland, OR, USA. 19-24 July 2008

Published: I I July 2008

BMC Neuroscience 2008, 9(SuppI I):PI43 doi:I0.I I86/I47I-2202-9-SI-PI43

This abstract is available from: http://www.biomedcentral.com/I47I-2202/9/SI/PI43

(c) 2008 Goedeke et al; licensee BioMed Central Ltd.

Synchronization of spiking activity in neuronal networks of the cortex has been proposed as a mechanism underlying higher brain functions. This idea is challenged by ongoing cortical activity generating large fluctuations in synaptic input, causing the neurons to operate in a noisy environment. The propagation of synchronized spiking in feed-forward subnetworks ("synfire chains") has been studied to demonstrate the feasibility of precise spike timing [1]. However, the theoretical analysis of even this toy model is impeded by the intricacy of calculating the distribution of spike times in response to time-dependent inputs. Therefore, most quantitative results rely on simulations or semi-numerical methods, and insight into the structure of the dynamics is limited. Recently [2], we showed that for a biophysically plausible integrate-andfire neuron model the probability of emitting a response spike is concentrated on the rising phase of the membrane potential transient caused by synchronous input and that during this time the instantaneous spiking rate is governed by the derivative of this transient. These observations were confirmed by an ad hoc calculation of the neuron's spike density. A corresponding instantaneous rate model enabled us to investigate the synchronization dynamics analytically. Inherent to the approach, the theory for the spike density breaks down at the peak of the membrane potential transient and during its descent. Meanwhile based on an exact series expression for the first passage time density of differentiable random processes (Wiener-Rice series) new successful approximations have been presented [3]. Here, we use the first term of the series to approximate the spike density of a stochastic integrateand-fire neuron model receiving time-dependent input.

The figure displays the densities of response spikes for four characteristic input parameters describing synchronous activity in feed-forward subnetworks (vertical: number of input spikes; horizontal: temporal dispersion of the input). Extending [2], the results of the new approximation (blue curves) are valid during the rising and the descending phase of the membrane potential transient and are in excellent agreement with the simulation results (gray curves). While in the earlier theory the relationship between output and input dispersion is independent of the amplitude of the input, the new theory resolves this degeneracy; it also holds in the less synchronized and subthreshold regime. We finally have a theory at hand covering the full dynamical range of synchronized spiking in cortical feed-forward networks. The approximation is valid for a wide range of relative time constants. In conclusion, our work provides a more fundamental theory for the earlier findings. The method has the potential to be applied to other time-dependent problems in computational neuroscience. 


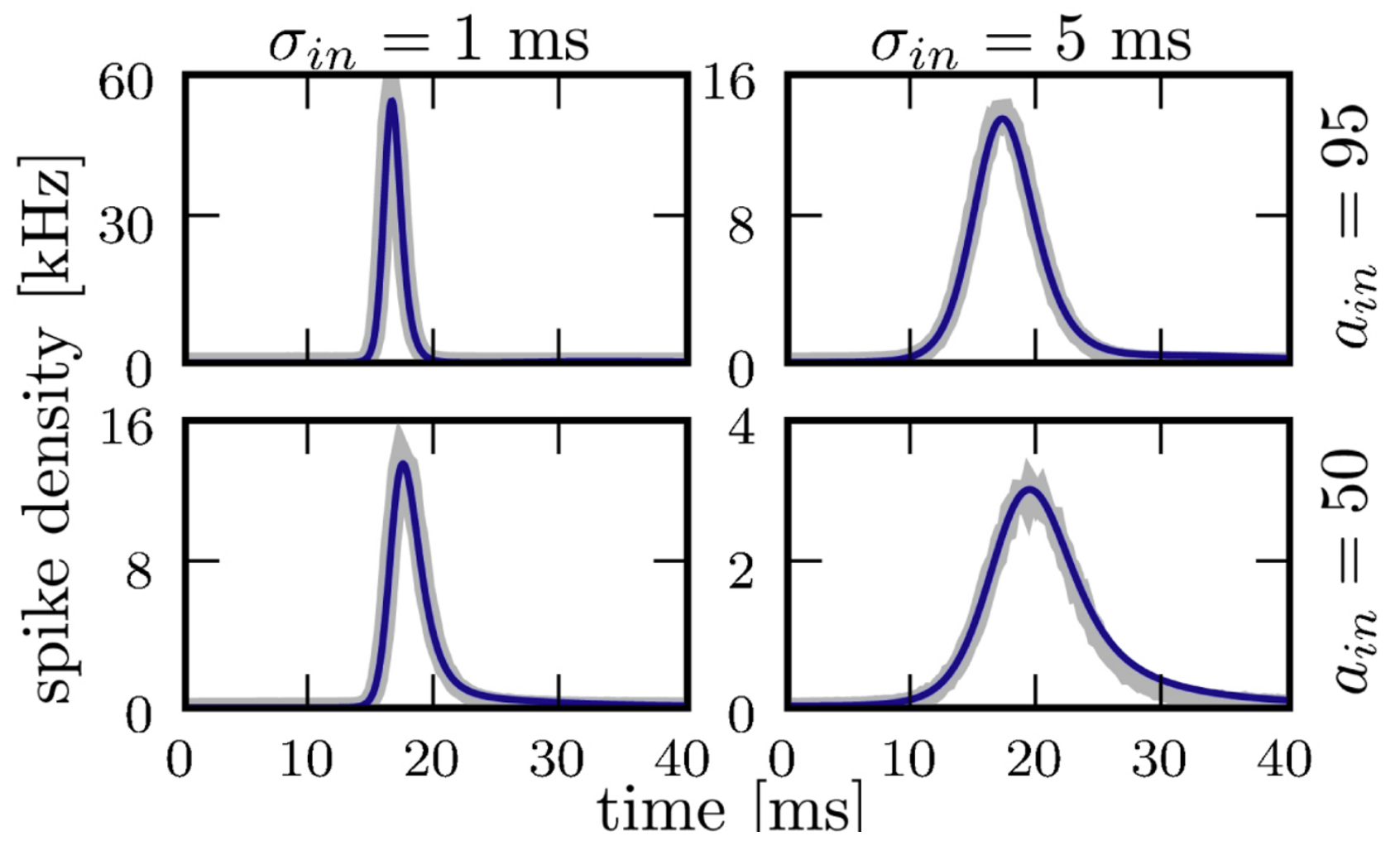

Figure I

Comparison of theoretical (blue) and simulated (gray) spike density of a population of leaky integrate-and-fire neurons in response to transient excitatory synaptic input caused by synchronous activity of presynaptic neurons. In the four panels, the number of input spikes decreases from top (95) to bottom (50) and their temporal precision from left (I ms) to right (5 ms). The vertical axes are individually scaled.

\section{Acknowledgements}

Partially funded by DIP FI.2, BMBF Grant 0IGQ0420 to the Bernstein Center for Computational Neuroscience Freiburg, EU Grant 15879 (FACETS), and German National Academic Foundation.

\section{References}

I. Diesmann M, Gewaltig MO, Aertsen A: Stable propagation of synchronous spiking in cortical neural networks. Nature 1999 , 402(676I):529-533.

2. Goedeke S, Diesmann M: The mechanism of synchronization in feed-forward neuronal networks. New J Phys 2008, 10:0 I5007.

3. Verechtchaguina T, Sokolov IM, Schimansky-Geier L: First passage time densities in resonate-and-fire models. Phys Rev E 2006, 73:03II08.

Publish with Biomed Central and every
scientist can read your work free of charge
"BioMed Central will be the most significant development for
disseminating the results of biomedical research in our lifetime. "
Sir Paul Nurse, Cancer Research UK
Your research papers will be:
• available free of charge to the entire biomedical community
• peer reviewed and published immediately upon acceptance
• cited in PubMed and archived on PubMed Central
• yours - you keep the copyright
Submit your manuscript here:
http://www.biomedcentral.com/info/publishing_adv.asp

